\title{
Evaluation of experimental calcium-containing primer in adhesive system on micro-tensile bond strength and acid resistance
}

\author{
Yukina OCHIAI ${ }^{1}$, Go INOUE ${ }^{1}$, Toru NIKAIDO ${ }^{1}$, Masaomi IKEDA² and Junji TAGAMI ${ }^{1}$ \\ ${ }^{1}$ Department of Cariology and Operative Dentistry, Division of Oral Health Sciences, Graduate School of Medical and Dental Sciences, Tokyo \\ Medical and Dental University (TMDU), 1-5-45 Yushima, Bunkyo-ku, Tokyo 113-8549, Japan \\ ${ }^{2}$ Department of Oral Prosthetic Engineering, Graduate School, Faculty of Dentistry, Tokyo Medical and Dental University (TMDU), 1-5-45 Yushima, \\ Bunkyo-ku, Tokyo 113-8549, Japan \\ Corresponding author, Go INOUE; E-mail: inoue.ope@tmd.ac.jp
}

\begin{abstract}
Modern adhesive systems have been developed for enhanced mechanical and antibacterial properties; however, no such systems contain a calcium source to enhance bond strength. Therefore, we evaluated the effect of an experimental calcium-containing primer $\left(10 \mathrm{wt} \% \mathrm{CaCl}_{2}\right)$ in two-step self-etching adhesive systems on micro-tensile bond strength $(\mu$-TBS) and acid resistance in dentin adhesive interface observations after acid-challenge using scanning electron microscopy. Using two types of primers and bonding agents (Clearfil SE Bond primer (SEP), experimental calcium-containing primer (CaP), Clearfil SE Bond (SEB), and Clearfil Protect Bond), we prepared four experimental groups. The $\mu$-TBS of CaP-SEB did not differ from that of SEP-SEB. Meanwhile, the CaP groups showed a thicker acid-base resistance zone. Thus, two-step self-etching adhesive system composed of experimental calciumcontaining primer can be used as an adhesive system with high acid resistance and acceptable bond strength.
\end{abstract}

Keywords: Acid resistance, Bond strength, Calcium, Self-etching adhesive

\section{INTRODUCTION}

In the development of the adhesive dentistry, technological changes (e.g., new adhesive systems and products, especially those with enhanced mechanical and antibacterial properties ${ }^{1)}$ are continually advancing. The success of restorations hinges on the adhesion stability between the resin composite and tooth structure. To maintain adhesion stability, the presence and quality of the hybrid layer is important ${ }^{2}$. In terms of the hybrid layer, self-etching adhesive systems create a thinner layer than phosphoric acid etching systems; however, there is less damage to the tooth structure, especially to dentin. The most widely used self-etching adhesive systems are two-step self-etching adhesives, which involve two application steps: the conditioning of tooth substrates with a self-etching primer, followed by the application of a bonding resin. Tsuchiya et al. . $^{3}$ observed inhibition of artificial secondary caries around restorations $<1-\mu \mathrm{m}$ thick beneath the hybrid layer (i.e., the acid-base-resistant zone (ABRZ)) only with self-etching adhesive systems. Their results suggest that self-etching primer in adhesive systems may be important in terms of the prevention of secondary caries around the restoration, sealing of restoration margins, and promotion of restoration durability. Self-etching adhesive systems contain specific functional monomers that determine adhesive performance. Among several functional monomers, 10-methacryloyloxydecyl dihydrogen phosphate (10-MDP) reportedly yields the most intensive chemical interaction with residual

Color figures can be viewed in the online issue, which is available at J-STAGE.

Received Aug 17, 2018: Accepted Oct 22, 2018

doi:10.4012/dmj.2018-266 JOI JST.JSTAGE/dmj/2018-266 hydroxyapatite compared with other monomers such as 2-methacryloxyethylphenyl hydrogen phosphate (Phenyl-P) and 4-methacyloxyethyl trimellitic acid, and its calcium salt appears to be the most hydrolytically stable based on its low dissolution rate in water ${ }^{4,5}$. As with 10-MDP-based two-step self-etch adhesive, Clearfil SE Bond (Kuraray Noritake Dental, Tokyo, Japan) has been proven to yield reliable results in terms of bonding effectiveness and durability ${ }^{6,7)}$, especially in terms of ABRZ formation at the adhesive interface when compared to other commercially available self-etching adhesive systems ${ }^{8}$.

Imazato et $a .^{9)}$ reported that usage of the antibacterial functional acidic monomer 12methacryloyloxydodecylpyridinium bromide (MDPB) effectively provided antibacterial effects in bonding systems. Clearfil Protect Bond (Kuraray Noritake Dental) is a bonding system that incorporates MDPB in the primer, as well as a fluoride-release source (surface-treated $\mathrm{NaF}$ ) in the bond. Several studies have reported that this adhesive system demonstrates high acid resistance and long-term bond durability ${ }^{10-13)}$. Thus, providing high functionality to the primer and/ or bonding agent has the potential to enhance adhesion and durability.

Teeth contain high concentrations of calcium and phosphate as hydroxyapatite, in which the proportion of calcium can reach as high as $39.98 \mathrm{wt} \%{ }^{14)}$. On the treated surface after removing caries, minerals, especially calcium ions, are lacking due to carious acid attack, and the mineral density decreases dramatically. As such, caries-affected dentin (CAD) has poor mechanical properties (e.g., bond strength) compared with normal 
tooth. Therefore, adhesive systems that can provide a calcium source and other minerals on the restorative interface could be advantageous.

As noted above, a high-functionality primer that contains an antibacterial monomer has been developed with the ability to promote good adhesion in clinical situations; however, no such primer systems include calcium, which might help to reinforce the adhesive interface, offering improved adhesion stability between the resin composite and tooth structure.

Therefore, the purpose of this study was to evaluate the influence of an experimental calcium-containing selfetching primer in a conventional two-step self-etching adhesive system in both fluoride-lacking and fluoridecontaining adhesive systems on dentin micro-tensile bond strength $(\mu$-TBS) and acid resistance.

\section{MATERIALS AND METHODS}

Human caries-free third molars were collected after obtaining written informed consent, and were stored frozen. It was approved by the human research ethics committee of Tokyo Medical and Dental University (No. D2013-022). For each tooth, the coronal portion was removed to expose a flat, midcoronal dentin surface using a low-speed diamond saw (Isomet, Buehler, Lake Bluff, IL, USA) under water refrigeration. The exposed dentin surface was ground using 600-grit silicon carbide abrasive paper under a water stream for $60 \mathrm{~s}$ to produce a standardized smear layer.

The basic composition of the experimental calcium-containing primer was Clearfil SE Bond primer supplemented with $10 \mathrm{wt} \% \mathrm{CaCl}_{2}$ (Table 1). It was prepared as a two-bottle-type primer to prevent calcium from combining with 10-MDP, allowing the calcium to exist in the ion form after mixing. The two bottles were mixed in equal proportions immediately before application to the restoration surface, where the application time and other procedures followed those of the Clearfil SE Bond primer.

In the bonding procedure, two types of primers (Clearfil SE Bond primer (SEP) and experimental calcium-containing primer $(\mathrm{CaP})$ ) and bonding agents (Clearfil SE Bond (SEB) without fluoride and Clearfil Protect Bond (PBB) with fluoride) were tested (Table 1), yielding a total of four experimental groups: SEP-SEB, SEP-PBB, CaP-SEB, and CaP-PBB. Each primer was applied on the dentin surface for $20 \mathrm{~s}$, after which the bonding agent was applied and light-cured for $10 \mathrm{~s}$ at a standardized distance of $8 \mathrm{~mm}$ using a halogen light curing unit (XL 3000, 3M, St. Paul, MN, USA) set at 600 $\mathrm{mW} / \mathrm{cm}^{2}$.

\section{$\mu$-TBS test}

Figure 1 presents a schematic representation of the experimental procedures for the $\mu$-TBS test. After completing the bonding procedure in each group, a resin composite (Clearfil AP-X, Shade A2, Kuraray Noritake

Table 1 Materials used in this study

\begin{tabular}{|c|c|c|c|}
\hline & Code & Material & Composition \\
\hline \multirow[b]{2}{*}{ Primer } & SEP & $\begin{array}{l}\text { Clearfil SE Bond primer } \\
(\mathrm{pH} 2.0)\end{array}$ & MDP, HEMA, hydrophilic dimethacrylate, water, initiator \\
\hline & $\mathrm{CaP}$ & $\begin{array}{l}\text { Experimental } \\
\text { calcium-containing primer } \\
(2 \text { Bottle, pH 2.4) }\end{array}$ & $\begin{array}{l}\text { Bottle A: MDP, HEMA, hydrophilic dimethacrylate, } \\
\text { water, initiator } \\
\text { Bottle B: } 10 \text { wt } \% \mathrm{CaCl}_{2} \text {, water, HEMA }\end{array}$ \\
\hline \multirow[b]{2}{*}{ Bond } & SEB & Clearfil SE Bond & $\begin{array}{l}\text { MDP, Bis-GMA, HEMA, CQ, hydrophobic dimethacrylate, } \\
\text { N-diethanol-p-toluidine, silanated colloidal silica }\end{array}$ \\
\hline & $\mathrm{PBB}$ & Clearfil Protect Bond & $\begin{array}{l}\text { MDP, Bis-GMA, HEMA, CQ, hydrophobic dimethacrylate, } \\
\text { N-diethanol-p-toluidine, silanated colloidal silica, } \\
\text { surface-treated NaF }\end{array}$ \\
\hline
\end{tabular}

MDP: 10-methacryloxydecyl dihydrogen phosphate, HEMA: 2-hydroxyethyl methacrylate, Bis-GMA: bisphenol-A-diglycidyl methacrylate, CQ: camphorquinone

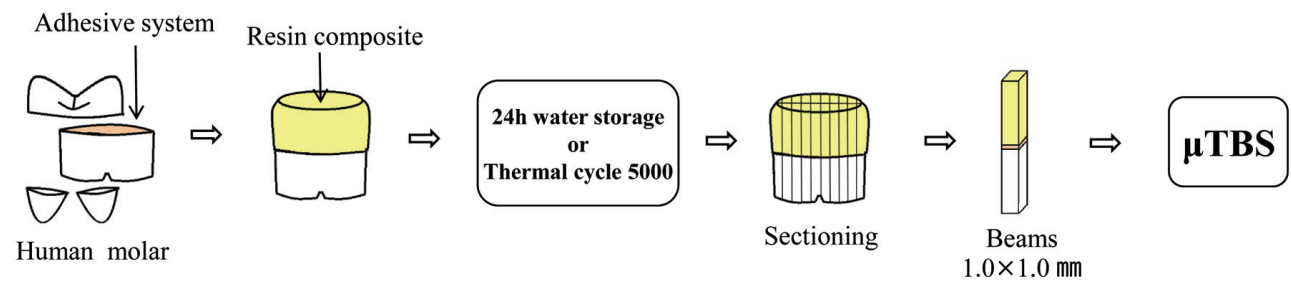

Fig. 1 Experimental procedure for the $\mu$-TBS test. 
Dental) was built up into an approximately 4-mm-thick layer on the treated dentin surface and light-cured for $40 \mathrm{~s}$. Before testing, the four experimental groups were further divided into a group stored in distilled water at $37^{\circ} \mathrm{C}$ for $24 \mathrm{~h}$ (24-h group) and a group treated with thermal cycling (Tokyo Giken, Tokyo, Japan) for 5,000 cycles between 5 and $55^{\circ} \mathrm{C}$ with a resting period of 30 s (TC5000 group). Then, each bonded specimen was longitudinally sectioned in both the buccal-lingual and mesiodistal directions across the bonded interface to obtain multiple sticks approximately $1.0 \times 1.0-\mathrm{mm}$ thick. Each stick was fixed to the attachment with cyanoacrylate adhesive (Model Repair II Blue, DentsplySankin, Tokyo, Japan), and tension stress was applied using a universal testing machine (EZ-SX, Shimadzu, Kyoto, Japan) at a crosshead speed of $1 \mathrm{~mm} / \mathrm{min}$ until failure.

The number of specimens differed among the groups (Table 2). Data indicated normal distribution by Shapiro-Wilk test $(p>0.05)$. However, the variance of data indicated non- homogeneous by Levene's test $(p<0.05)$. Therefore, the mean bond strength was analyzed by two-way analysis of variance and Dunnett's T3 test. In addition, the mode of failure of debonded specimens was evaluated under scanning electron microscopy (SEM; JSM-5310LV, JEOL, Tokyo, Japan) and classified into one of four categories: 1) cohesive failure in resin or mixed failure between resin and adhesive; 2) interfacial failure between adhesive and dentin; 3) mixed failure between adhesive and dentin; and 4) cohesive failure in dentin ${ }^{15}$. The frequency of failure mode was analyzed with the Fisher exact test. All statistical analyses were performed at the 0.05 significance level using the statistical software SPSS ver. 22 (IBM, Armonk, NY, USA).

\section{ABRZ observation}

Figure 2 illustrates the specimen preparation for the ABRZ observations. For each group, three molars were sectioned into 2-mm-thick dentin disks at the midcoronal portion horizontal to the tooth axis. Each primer was applied on the dentin surface for $20 \mathrm{~s}$ and bonding agent was applied and light-cured (XL 3000, 3M) for $10 \mathrm{~s}$. Then, Estelite flow quick (Shade A2, Tokuyama Dental, Tokyo, Japan) was placed between pairs of treated dentin disks and light-cured for $40 \mathrm{~s}$ from the top and bottom surfaces to form a dentin disk sandwich ${ }^{3)}$. After 24-h storage or TC5000 treatment, samples for ABRZ observation were embedded in epoxy resin (Epoxicure 2, Buehler). Then, the acid-base challenge was performed

Table 2 The mean micro-tensile bond strengths of 24-h and TC5000 (MPa)

\begin{tabular}{clc}
\hline Group & \multicolumn{1}{c}{24 -h } & TC5000 \\
\hline SEP-SEB & $53.8 \pm 7.1^{\mathrm{a}}(n=18)$ & $72.9 \pm 8.3^{\mathrm{b}}(n=20)$ \\
SEP-PBB & $55.7 \pm 7.1^{\mathrm{aA}}(n=24)$ & $56.2 \pm 5.7^{\mathrm{A}}(n=25)$ \\
CaP-SEB & $55.7 \pm 7.7^{\mathrm{a}}(n=22)$ & $68.1 \pm 6.4^{\mathrm{b}}(n=18)$ \\
CaP-PBB & $47.5 \pm 6.0^{\mathrm{aB}}(n=18)$ & $45.6 \pm 6.0^{\mathrm{B}}(n=23)$ \\
\hline
\end{tabular}

Values are given as mean $\pm \mathrm{SD}$ in $\mathrm{MPa}$ (number of specimens). Same small letter in same column means no significant difference $(p>0.05)$. Same capital letter in same raw means no significant difference $(p>0.05)$. SEP: Clearfil SE Bond primer, SEB: Clearfil SE Bond, CaP: Experimental calcium-containing primer, PBB: Clearfil Protect Bond

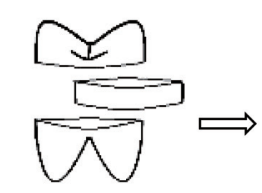

Human molar

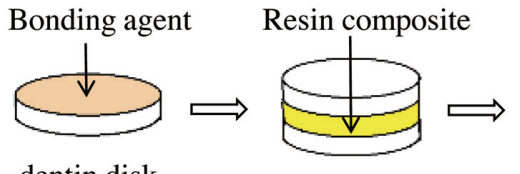

dentin disk

Acid-base challenge

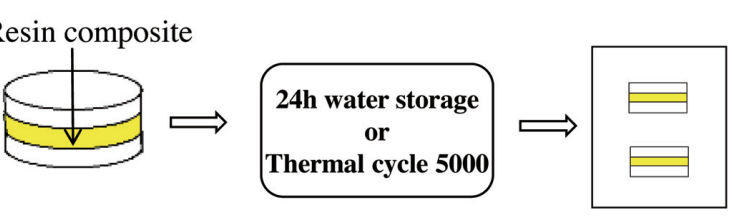

Embedding

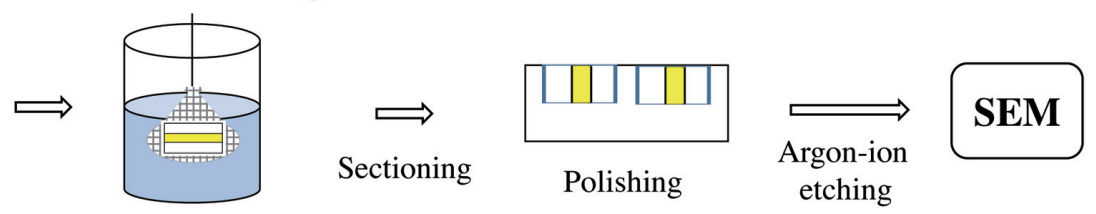

(1)Demineralizing solution (pH4.5) $90 \mathrm{~min}$

(2) $6 \% \mathrm{NaOCl} 20 \mathrm{~min}$

Fig. 2 Specimen preparation for the ABRZ observations. 
in demineralizing solution $\left(2.2 \mathrm{mM} \mathrm{CaCl}_{2}, 2.2 \mathrm{mM}\right.$ $\mathrm{Na}_{2} \mathrm{HPO}_{4}, 50 \mathrm{mM}$ acetic acid; pH 4.5) for $90 \mathrm{~min}$, after which the samples were immersed in $5 \% \mathrm{NaOCl}$ for $20 \mathrm{~min}$ to remove any exposed demineralized dentin collagen fibrils. Then, 4-META/MMA-TBB Resin (Super Bond C\&B, Sun Medical, Shiga, Japan) was placed on the demineralized surface to protect the observed surface. Specimens were sectioned into 2-mm-thick slabs and polished with silicon carbide papers up to 1200 grit, followed by diamond paste (Struers, Copenhagen, Denmark) up to $0.25 \mu \mathrm{m}$. Next, the polished surfaces were etched with an argon-ion beam (EIS-IE, Elionix, Tokyo, Japan) at $1 \mathrm{kV}, 0.2 \mathrm{~mA} / \mathrm{cm}^{2}$ for $30 \mathrm{~s}$ to highlight the hybrid layer. After coating observed surface with gold, the ABRZ of the dentin adhesive interface was observed by SEM (S-4000, Hitachi, Tokyo, Japan) with an accelerating voltage of $15 \mathrm{kV}$.

\section{RESULTS}

$\mu-T B S$ test

The mean values of the $\mu$-TBS test (MPa) (24-h/TC5000)

Table 3 Failure mode of debonded specimens

\begin{tabular}{ccc}
\hline Group & $24-\mathrm{h}$ & TC5000 \\
\hline SEP-SEB & {$[11 / 50 / 17 / 22]^{\mathrm{aA}}$} & {$[5 / 20 / 20 / 55]^{\mathrm{A}}$} \\
SEP-PBB & {$[0 / 71 / 0 / 29]^{\mathrm{aB}}$} & {$[0 / 68 / 16 / 16]^{\mathrm{B}}$} \\
CaP-SEB & {$[0 / 54 / 23 / 23]^{\mathrm{a}}$} & {$[11 / 17 / 22 / 50]$} \\
CaP-PBB & {$[0 / 67 / 11 / 22]^{\mathrm{aC}}$} & {$[4 / 60 / 18 / 18]^{\mathrm{bC}}$} \\
\hline
\end{tabular}

Percentage of failure mode [cohesive failure in resin or mixed failure between resin and adhesive/interfacial failure between adhesive and dentin/mixed failure between adhesive and dentin/cohesive failure in dentin]. Same small letter in same column means no significant difference $(p>0.05)$. Same capital letter in same raw means no significant difference $(p>0.05)$. SEP: Clearfil SE Bond primer, SEB: Clearfil SE Bond, CaP: Experimental calcium-containing primer, PBB: Clearfil Protect Bond
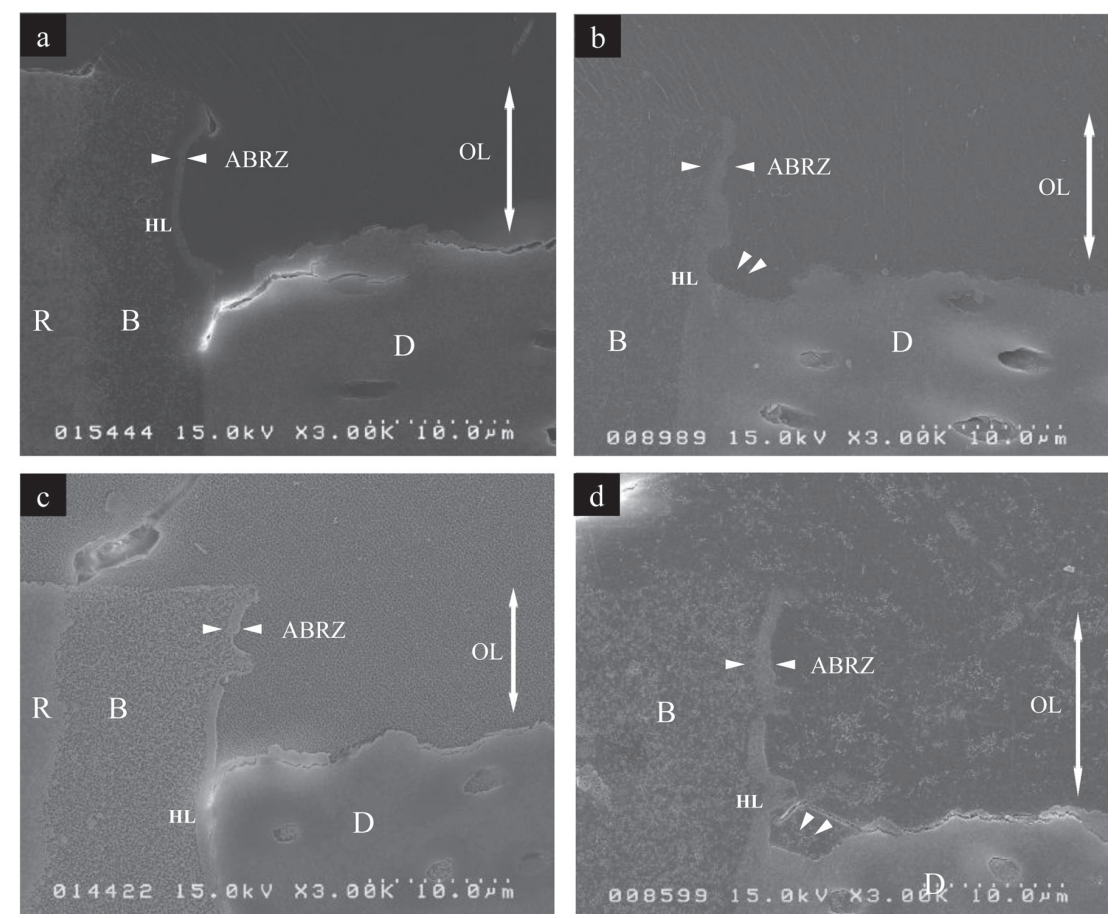

Fig. 3 SEM images $(\times 3,000)$ of the ultrastructure of the interfaces after acid-base challenge in four experimental groups after 24 -h of storage in water.

(a) SEP-SEB: A thin ABRZ of approximately $0.25 \mu \mathrm{m}$ (arrows) was observed beneath the hybrid layer, with a thicker hybrid layer compared to the other groups. (b) SEP-PBB: A thick ABRZ $>1.0-\mu \mathrm{m}$ wide with slopes from the top of the outer lesion (arrows) was observed near the hybrid layer. (c) CaP-SEB: An ABRZ $>0.5-\mu m$ wide (arrows) was observed, with no clear hybrid layer compared to SEP-SEB. (d) CaP-PBB: A thick ABRZ >1.0- $\mu$ m wide with slopes (arrows) was observed. R: Resin, B: Bonding, OL: Outer lesion, D: Dentin, ABRZ: Acid based resistance zone 

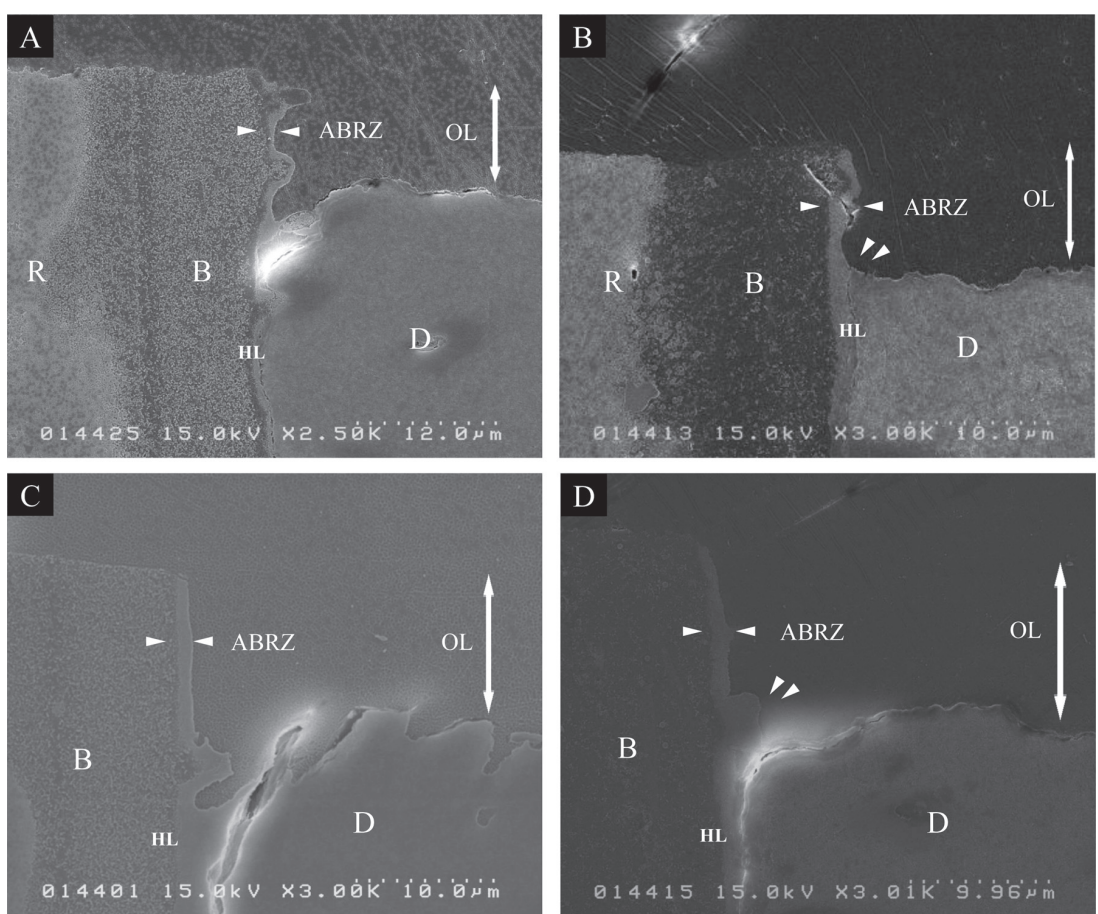

Fig. 4 SEM images $(\times 2,500-3,000)$ of the ultrastructure of the interfaces after the acid-base challenge in the TC5000 groups.

There were no characteristic differences before and after the thermal cycle. (A) SEP-SEB, (B) SEP-PBB, (C) CaPSEB, (D) CaP-PBB. R: Resin, B: Bonding, OL: Outer lesion, D: Dentin, ABRZ: Acid based resistance zone.

are shown in Table 2. In the 24-h storage group, CaP-PBB exhibited the lowest bond strength; however, there was no significant difference among the other 24 -h groups $(p>0.05)$. After thermal cycling, the SEP-SEB and CaPSEB groups showed significantly higher bond strengths than before thermal cycling, while the other two groups showed no significant difference. In the TC5000 group, SEP-SEB and CaP-SEB showed significantly higher bond strengths than the other two groups, whereas CaPPBB exhibited the lowest among all groups.

Table 3 presents the results of the failure mode analysis. In all 24-h groups, interfacial failure between adhesive and dentin was mainly observed, with no significant differences $(p>0.05)$. In the TC5000 groups, the SEP-SEB and SEP-PBB groups showed significant difference in the frequencies of interfacial failure and cohesive failure in dentin, and the CaP-SEB group showed a significant difference in the frequency of interfacial failure $(p<0.05)$. Comparing the groups before and after thermal cycling, there was a tendency for the rate of interfacial failure to decrease and cohesive failure in dentin to increase in both the SEP-SEB and CaPSEB groups; however, only the CaP-SEB group showed a significant difference in the rate of interfacial failure $(p<0.05)$, whereas the other groups showed no significant differences before and after thermal cycling $(p>0.05)$.

\section{ABRZ observation}

Figures 3 and 4 present SEM images of the adhesive- dentin interface after acid-base challenge. ABRZs were clearly observed beneath the hybrid layer in all specimens. There were no characteristic differences before and after TC5000 treatment; however, the morphological features of the ABRZ differed among the groups. Sloped ABRZ formations were predominantly observed in the SEP-PBB and CaP-PBB groups. Meanwhile, the SEB-SEP and CaP-SEB groups showed erosion along the top of the outer lesion. Interestingly, the ABRZ of the CaP-SEB group was thicker than that of the SEP-SEB group. Finally, the SEP-SEB group showed the thinnest ABRZ, although it had the greatest hybrid layer width.

\section{DISCUSSION}

\section{$\mu$-TBS results}

In the 24-h storage group, CaP-SEB did not show reduced bond strength compared to the other groups. Burrow et al. ${ }^{16)}$ reported that application of $10 \%$ citric acid in $20 \% \mathrm{CaCl}_{2}$ conditioner before applying the bonding system (Liner Bond, Kuraray Noritake Dental) yielded a high bond strength after $24 \mathrm{~h}$ of storage. In this study, $\mathrm{CaCl}_{2}$ was used as a calcium source in the experimental primer. Normally, including additional ingredients and increasing the $\mathrm{pH}$ can affect the bonding ability. However, the materials and conditions in their study differed from those in this study, which could explain why $\mathrm{CaCl}_{2}$ did not affect the early bond strength in the 


\section{CaP-SEB group.}

Clearfil Protect Bond has been shown to have a lower early bond strength than Clearfil SE Bond ${ }^{11)}$. Moreover, adhesive systems incorporating MDPB showed slightly, albeit non-significantly, lower bond strength than adhesive systems without $\mathrm{MDPB}^{9,10)}$. In this study, the early bond strength in the SEP-PBB group was the same as those of the other groups. This was possibly because we used Clearfil SE Bond primer (containing 10-MDP) instead of Clearfil Protect Bond primer (containing MDPB) in this study.

Among the groups, the CaP-PBB group showed the lowest bond strength. Calcium, which remained in its ion form on the dentin surface, may have combined with fluoride ions from the adhesive to form $\mathrm{CaF}_{2}$ or other calcium-fluoride complexes, acting as an inhibitor of bond strength on the surface of dentin by inhibiting the penetration of the bonding agent.

A previous study reported an experimental adhesive system based on Clearfil SE Bond with primer containing $10 \mathrm{wt} \% \mathrm{CaCl}_{2}$ and dentin matrix protein along with bonding agent containing hydroxyapatite, which showed significantly decreased dentin bond strength ${ }^{17)}$. However, the calcium-containing primer system in this study did not affect the bond strength in the absence of fluoride. Thus, this condition may have affected the results.

After TC5000 treatment, the SEB groups exhibited significantly higher bond strengths than those before TC5000 treatment, whereas the PBB groups showed no significant differences. It was reported that the bond strength of Clearfil SE Bond primer increased significantly after 10,000 thermal cycles $^{18)}$, although there was no significant difference in the $\mu$-TBS of Clearfil Protect Bond before and after 20,000 thermal cycles ${ }^{19)}$. From the results of the failure mode, the statistical analysis of all failure modes did not support the results of the bond strength test. However, the SEB groups showed a tendency toward a lower interfacial failure rate and increased cohesive failure rate after thermal cycling.

These results suggest that post-curing of incomplete polymerization within the resin composite and adhesive may be caused by thermal loading when using Clearfil SE Bond, while fluoride-containing bond may not promote this reaction. Meanwhile, other studies reported that the bond strength of Clearfil Protect Bond was increased after long-term storage in water ${ }^{11,13)}$. Therefore, longer thermal cycle or water storage tests may be required to effectively evaluate bond durability.

As a potential factor of the bond-strength increase in the CaP-SEB group, we considered the effects of the MDP-calcium salt. Adhesive containing the functional monomer 10-MDP, which effectively interacts chemically with hydroxyapatite within a clinically reasonable time, and which exhibits minimal solubility as the calcium salt, showed no signs of degradation in terms of bond strength and interfacial ultrastructure ${ }^{5}$. In addition, the calciumcontaining primer may have promoted the formation of the MDP-calcium salt at the dentin interface, which may have resulted in the increased bond strength of the
CaP-SEB group after thermal loading.

\section{ABRZ observation}

According to the acid-challenge test results, the SEPSEB group had a thinner ABRZ than the other groups. In addition, slope formation at the bottom of the ABRZ was only observed in the fluoride bond (PBB) group, and not in the CaP-SEB or SEP-SEB groups. Moreover, the CaP groups had a thicker ABRZ than the SEP groups. These results suggest that the presence of calcium may influence the thickness and quality of the ABRZ, and its function against acids may differ from that of fluoride adhesive.

Shinohara et al. ${ }^{10)}$ reported similar ABRZ characteristics, with no slope formation observed in the Clearfil SE primer (10-MDP, pH=2.0) with Clearfil SE bond group and Clearfil Protect Bond primer (MDBP, $\mathrm{pH}=2.0$ ) with Clearfil SE Bond group, and slope formation was only observed in fluoride-containing bond groups. These results suggest that slope formation may depend on the existence of fluoride, not the $\mathrm{pH}$ of the primers or functional monomers. Moreover, slope formation and a thicker ABRZ were observed in the fluoride-containing adhesive. The authors speculated that the presence of a fluoride-releasing component in the adhesive resin prevented demineralization of the dental structure against acid $\operatorname{attack}^{8)}$.

The calcium-containing primer had a higher $\mathrm{pH}$ (2.4) than the Clearfil SE primer (2.0) and milder etching effect. The etching aggressiveness of self-etching adhesive systems can be classified as strong $(\mathrm{pH}<1)$, intermediately strong $(\mathrm{pH} \approx 1.5)$, mild $(\mathrm{pH} \approx 2)$, and ultramild $(\mathrm{pH} \geq 2.5)^{20)}$. Mild self-etching partially removes the smear layer, forming a thin hybrid layer ${ }^{21)}$. Koshiro et $a l .{ }^{22)}$ reported that hydroxyapatite crystals remained around collagen fibrils even after self-etching treatment because of their relatively high $\mathrm{pH}(>2)$. Meanwhile, ultra-mild self-etching can only expose superficial dentin collagen and to produce the characteristic structure called the nanometer interaction zone (NIZ), which has a thinner and less-demineralized interface, with the presence of hydroxyapatite-coated collagen fibril ${ }^{22)}$. The interface of the NIZ is expected to be stronger and more durable because of its lack of complete exposure of collagen fibers compared with the typical hybrid layer of mild etching. The calcium-containing primer $(\mathrm{pH}=2.4)$ in this study exhibited ultra-mild etching properties $(\mathrm{pH} \geq 2.5)$; therefore, it is suspected that hydroxyapatite in collagen fibrils, which can react with functional monomers, remained within the hybrid layer, resulting in the thicker ABRZ.

As another factor, the calcium salt of 10-MDP is not readily soluble, and less-soluble calcium salts of acidic molecules ${ }^{4}$ result in more intense and stable molecular adhesion to hydroxyapatite-based substrates according to the adhesion-decalcification concept ${ }^{23)}$. For example, Matsui et al. ${ }^{24)}$ revealed that the presence of 10 -MDP in adhesive contribute to reinforce dentin against acid attack. As another hypothesis explaining the thick $\mathrm{ABRZ}$ in the CaP groups, we speculated that more 10 - 
MDP salt was produced in the etched dentin surface due to the presence of calcium in the primer, increasing acid resistance.

The CaP-PPB showed improved acid resistance due to the thickness of the ABRZ with slope formation. Thus, the calcium in the primer appeared to work cooperatively with the fluoride in the bond, increasing the acid resistance. The CaP-PBB group also showed the lowest bond strength. Similarly, studies have reported that some types of adhesive systems used in clinical situations show lower bond strengths than CaP-PBB under similar conditions as those in this study ${ }^{25}$. Given its advantage in acid resistance, we suggest the use of this combination as a new bonding system.

In this study, only intact dentin was used as the adherent, although clinically resin composite restoration may be applied to either sound dentin or CAD. However, as indicated by several studies, there are differences in the mechanical and chemical properties between intact dentin and $\mathrm{CAD}^{26,27)}$, where increasing levels of demineralization result in poorer mechanical properties and reduced mineral contents ${ }^{28}$. It is expected that calcium-containing adhesive could overcome such weaknesses via the remineralization of CAD. To consider this application clinically, the evaluation of calciumcontaining primer on $\mathrm{CAD}$ is required.

\section{CONCLUSION}

The results suggest that calcium in primer does not reduce the bond strength compared with normal primer and improves the acid resistance of bonding systems. Thus, calcium-containing primer with fluoride bond is expected as new bonding system to improve the chemical properties of the bond. Regardless, further studies to evaluate the effects of calcium-containing primer on $\mathrm{CAD}$ and more thermal cycle load or storage tests are required to better evaluate its durability. Overall, we suggest that a two-step self-etching adhesive system composed of experimental calcium-containing primer can be used as an adhesive system with high acid resistance and acceptable bond strength.

\section{ACKNOWLEDGMENTS}

This study was supported in part by a Grant-in-Aid for Scientific Research (No. 26861590) from the Japanese Ministry of Education, Culture, Sports, Science, and Technology. We wish to thank Kuraray Noritake Dental and Sun Medical for providing the materials required for this study.

\section{REFERENCES}

1) Miyazaki M, Tsujimoto A, Tsubota K, Takamizawa $\mathrm{T}$, Kurokawa H, Platt JA. Important compositional characteristics in the clinical use of adhesive systems. J Oral Sci 2014; 56: 1-9.

2) Nakabayashi N, Nakamura M, Yasuda N. Hybrid layer as a dentin-bonding mechanism. J Esthet Dent 1991; 3: 133-138.

3) Tsuchiya S, Nikaido T, Sonoda H, Foxton RM, Tagami J.
Ultrastructure of the dentin-adhesive interface after acidbase challenge. J Adhes Dent 2004; 6: 183-190.

4) Yoshida Y, Nagakane K, Fukuda R, Nakayama Y, Okazaki M, Shintani H, et al. Comparative study on adhesive performance of functional monomers. J Dent Res 2004; 83: 454-458.

5) Inoue S, Koshiro K, Yoshida Y, De Munck J, Nagakane K, Suzuki K, et al. Hydrolytic stability of self-etch adhesives bonded to dentin. J Dent Res 2005; 84: 1160-1164.

6) Toledano M, Osorio R, de Leonardi G, Rosales-Leal JI, Ceballos L, Cabrerizo-Vilchez MA. Influence of self-etching primer on the resin adhesion to enamel and dentin. Am J Dent 2001; 14: 205-210

7) Tsujimoto A, Barkmeier WW, Takamizawa T, Watanabe H, Johnson WW, Latta MA, et al. Comparison between universal adhesives and two-step self-etch adhesives in terms of dentin bond fatigue durability in self-etch mode. Eur J Oral Sci 2017; 125: 215-222.

8) Inoue G, Nikaido T, Foxton RM, Tagami J. The acid-base resistant zone in three dentin bonding systems. Dent Mater 2009; 28: 717-721.

9) Imazato S, Kinomoto $Y$, Tarumi H, Ebisu S, Tay FR. Antibacterial activity and bonding characteristics of an adhesive resin containing antibacterial monomer MDPB. Dent Mater 2003; 19: 313-319.

10) Shinohara MS, Yamauti M, Inoue G, Nikaido T, Tagami J, Giannini M, et al. Evaluation of antibacterial and fluoridereleasing adhesive system on dentin - microtensile bond strength and acid-base challenge. Dent Mater J 2006; 25: $545-552$.

11) Shinohara MS, De Goes MF, Schneider LF, Ferracane JL, Pereira PN, Di Hipólito V, et al. Fluoride-containing adhesive: durability on dentin bonding. Dent Mater 2009; 25: 13831391.

12) Ichikawa C, Nikaido T, Inoue G, Sadr A, Tagami J. Ultramorphological evaluation of the dentin acid-base resistant zone of two-step self-etching systems after longterm storage in water. J Adhes Dent 2012; 14: 207-213.

13) Khoroushi M, Rafiei E. Effect of thermocycling and water storage on bond longevity of two self-etch adhesives. Gen Dent 2013; 61: 39-44.

14) Pugach MK, Strother J, Darling CL, Fried D, Gansky SA, Marshall SJ, et al. Dentin caries zones: mineral, structure, and properties. J Dent Res 2009; 88: 71-76.

15) Atomura J, Inoue G, Nikaido T, Yamanaka K, Uo M, Tagami J. Influence of FCP-COMPLEX on bond strength and the adhesive-artificial cariesaffected dentin interface. Dent Mater J 2018; 375: 775-782.

16) Burrow MF, Tagami J, Negishi T, Nikaido T, Hosoda H. Early tensile bond strengths of several enamel and dentin bonding systems. J Dent Res 1994; 73: 522-528.

17) Shinkai K, Taira Y, Suzuki M, Kato C, Yamauchi J, Suzuki S, et al. Dentin bond strength of an experimental adhesive system containing calcium chloride, synthetic peptides derived from dentin matrix protein 1 ( $\mathrm{pA}$ and $\mathrm{pB})$, and hydroxyapatite for direct pulp capping and as a bonding agent. Odontology 2010; 98: 110-116.

18) Tsujimoto A, Barkmeier WW, Takamizawa T, Latta MA, Miyazaki M. Bonding performance and interfacial characteristics of short fiber-reinforced resin composite in comparison with other composite restoratives. Eur J Oral Sci 2016; 124: 301-308.

19) De Munck J, Van Landuyt K, Coutinho E, Poitevin A, Peumans M, Lambrechts P, et al. Micro-tensile bond strength of adhesives bonded to Class-I cavity-bottom dentin after thermo-cycling. Dent Mater 2005; 21: 999-1007.

20) Van Meerbeek B, Peumans M, Poitevin A, Mine A, Van Ende A, Neves A, et al. Relationship between bond-strength tests and clinical outcomes. Dent Mater 2010; 26: e100-121.

21) Giannini M, Makishi P, Ayres AP, Vermelho PM, Fronza 
BM, Nikaido T, et al. Self-etch adhesive systems: A literature review. Braz Dent J 2015; 26: 3-10.

22) Koshiro K, Sidhu S, Inoue S, Ikeda T, Sano H. New concept of resin-dentin interfacial adhesion: The nanointeraction zone. J Biomed Mater Res B Appl Biomater 2006; 77: 401-408.

23) Yoshida Y, Van Meerbeek B, Nakayama Y, Yoshioka M, Snauwaert J, Abe Y, et al. Adhesion to and decalcification of hydroxyapatite by carboxylic acids. J Dent Res 2001; 80: 1565-1569.

24) Matsui N, Takagaki T, Sadr A, Ikeda M, Ichinose S, Nikaido $\mathrm{T}$, et al. The role of MDP in a bonding resin of a two-step selfetching adhesive system. Dent Mater J 2015; 34: 227-233.

25) Mallick R, Sarangi P, Mohanty S, Behera S, Nanda S, Satapathy SK. Micro-tensile bond strength of different adhesive systems on sound dentin and resin-based composite: An in-vitro study. J Conserv Dent 2015; 18: 379-383.

26) Inoue G, Tsuchiya S, Nikaido T, Foxton RM, Tagami J. Morphological and mechanical characterization of the acidbase resistant zone at the adhesive-dentin interface of intact and caries-affected dentin. Oper Dent 2006; 31: 466-472.

27) Shibata S, Vieira LC, Baratieri LN, Fu J, Hoshika S, Matsuda $\mathrm{Y}$, et al. Evaluation of microtensile bond strength of selfetching adhesives on normal and caries-affected dentin. Dent Mater J 2016; 35: 166-173.

28) Bolte M, Krefting ER, Clemen G. Hard tissue of teeth and their calcium and phosphate content in Ambystoma mexicanum (Urodela: Ambystomatidae). Ann Anat 1996; 178: 71-80. 